\title{
Interstitial Photodynamic Therapy
}

National Cancer Institute

\section{Source}

National Cancer Institute. Interstitial Photodynamic Therapy. NCI Thesaurus. Code C102743.

Photodynamic therapy that uses light-emitting fibers to treat deep-seated solid tumors. 